Philippe Souplet

\title{
Single-point blow-up for a semilinear parabolic system
}

Received December 16, 2006 and in revised form April 11, 2007

Abstract. We consider positive solutions of the system

$$
u_{t}-\Delta u=v^{p}, \quad v_{t}-\Delta v=u^{q}
$$

in a ball or in the whole space, with $p, q>1$. Relatively little is known on the blow-up set for semilinear parabolic systems and, up to now, no result was available for this basic system except for the very special case $p=q$. Here we prove single-point blow-up for a large class of radial decreasing solutions. This in particular solves a problem left open in a paper of A. Friedman and Y. Giga (1987). We also obtain lower pointwise estimates for the final blow-up profiles.

\section{Introduction and main results}

Let us consider the following reaction-diffusion system:

$$
\begin{cases}u_{t}-\Delta u=|v|^{p-1} v, & x \in \Omega, t>0, \\ v_{t}-\Delta v=|u|^{q-1} u, & x \in \Omega, t>0, \\ u=v=0, & x \in \partial \Omega, t>0, \\ u(x, 0)=u_{0}(x), & x \in \Omega, \\ v(x, 0)=v_{0}(x), & x \in \Omega,\end{cases}
$$

where $p, q>1, \Omega$ is a smooth domain in $\mathbb{R}^{n}$, and $u_{0}, v_{0} \in L^{\infty}(\Omega)$. We denote by $T=$ $T\left(u_{0}, v_{0}\right) \in(0, \infty]$ the maximal existence time of the classical solution $(u, v)$ of problem (1.1). We shall mainly consider the case when $\Omega$ is a ball $B_{R}=\left\{x \in \mathbb{R}^{n} ;|x|<R\right\}$ and solutions are radially symmetric, i.e. depend only on $r=|x|$ at a given time $t$. Note that this is the case whenever $u_{0}, v_{0}$ are radially symmetric. When no confusion is likely, we shall identify $(u(x, t), v(x, t))$ and $(u(r, t), v(r, t))$. If, moreover, $u_{0}$ and $v_{0}$ are radially decreasing, then $u_{r}, v_{r} \leq 0$ in $[0, R] \times(0, T)$. It is well-known $[12,13]$ that $T<\infty$ when the initial data are suitably large. Throughout the paper, we shall use the notation

$$
\alpha=\frac{p+1}{p q-1}, \quad \beta=\frac{q+1}{p q-1}
$$

for the standard scaling exponents of system (1.1).

Ph. Souplet: Université Paris 13, CNRS, UMR 7539 LAGA 93430, Villetaneuse, France; e-mail: souplet@math.univ-paris13.fr

Mathematics Subject Classification (2000): Primary 35K50, 35B40; Secondary 35K55, 35K57 
In the case of a single equation, the blow-up set was first studied in [24], where the first result on single-point blow-up was obtained for $n=1, p$ large and particular initial data. It was next shown in [10] that when $\Omega=B_{R}$ and $p>1$, single-point blow-up occurs whenever the solution is positive, radially symmetric and nonincreasing as a function of $r$. See $[10,18,3,22]$ for further results in the scalar case.

As for system (1.1), little is known concerning the blow-up set. As far as we know, the only available result is that of [9]. In that work, single-point blow-up was proved for $n=1$ and symmetric decreasing solutions under the very restrictive condition that $p=q$, but the question for $p \neq q$ was left open. The purpose of the present paper is to solve this problem. Our main result is the following:

Theorem 1. Let $p, q>1$ and $\Omega=B_{R}$. Let $(u, v)$ be a radially symmetric, nonnegative classical solution of (1.1) such that $u_{r}, v_{r} \leq 0$ and $T<\infty$. Assume that $(u, v)$ satisfies the upper blow-up estimates

$$
\sup _{0<t<T}(T-t)^{\alpha}\|u(t)\|_{\infty}<\infty, \quad \sup _{0<t<T}(T-t)^{\beta}\|v(t)\|_{\infty}<\infty .
$$

In particular, (1.3) is known to be true if we assume in addition that either

$$
u_{t}, v_{t} \geq 0 \quad \text { in } B_{R} \times(0, T) \text {, }
$$

or

$$
\max (\alpha, \beta) \geq n / 2 \text {. }
$$

Then blow-up occurs only at the origin, i.e.

$$
\sup _{0<t<T}(u(r, t)+v(r, t))<\infty \quad \text { for any } r \in(0, R) .
$$

Remarks 1.1. (i) Estimate (1.3) under assumption (1.4) is due to [5], where it was actually proved for any bounded domain. Property (1.4) is true whenever $u_{0}, v_{0} \in L^{\infty} \cap H^{2} \cap$ $H_{0}^{1}(\Omega)$ satisfy $\Delta u_{0}+v_{0}^{p} \geq 0, \Delta v_{0}+u_{0}^{q} \geq 0$. As for (1.3) under assumption (1.5), this follows from straightforward modifications of the proof in [4] (where this was shown for $\Omega=\mathbb{R}^{n}$ but without symmetry restrictions). We also refer to $[2,1,8,21]$ for other results related to property (1.3).

(ii) The result of Theorem 1 remains true for the Cauchy problem (that is, $R=\infty$ ) provided $u_{0}, v_{0}$ are not both constant. This follows from straightforward modifications of the proof.

On the other hand, we establish pointwise lower bounds on the blow-up profile for a suitable class of solutions.

Theorem 2. Let $p, q>1$ and $\Omega=B_{R}$. Let $(u, v)$ be a radially symmetric, nonnegative classical solution of (1.1) satisfying $u_{r}, v_{r} \leq 0$ and $u_{t}, v_{t} \geq 0$, and such that $T<\infty$. Then we have the estimates

$$
|x|^{2 \alpha} u(x, T) \geq c_{1}, \quad 0<|x|<\eta,
$$


and

$$
|x|^{2 \beta} v(x, T) \geq c_{2}, \quad 0<|x|<\eta
$$

for some $c_{1}, c_{2}, \eta>0$.

Remarks 1.2. (i) In the scalar case, for radially decreasing solutions, the upper bound $u(x, t) \leq C_{\varepsilon}|x|^{-2 /(p-1)-\varepsilon}, \varepsilon>0$, is known [10]. More precise estimates are available in the case $p<(n+2) /(n-2)_{+}$(see [17, 23]). For system (1.1), the upper bounds complementing (1.6) and (1.7) remain an open problem.

(ii) Theorem 2 partially improves on [19, Theorem 1], where it was shown (without symmetry or monotonicity restrictions on the solution) that $\limsup _{t \rightarrow T}\|u(t)\|_{r_{1}}=$ $\lim \sup _{t \rightarrow T}\|u(t)\|_{r_{2}}=\infty$ for any $r_{1}>n /(2 \alpha)$ and $r_{2}>n /(2 \beta)$.

Besides the results already cited, let us mention that some fine asymptotic properties of blow-up solutions for system (1.1) have been studied in [1] and [25] from a different point of view. In particular, these articles contain a classification and sharp estimates of blow-up profiles, but the results are obtained under the assumption that $|p-q| \ll 1$ (due to certain compactness arguments depending on some known results in the scalar case). However, even under this assumption, no examples of single-point blow-up are given there. For other aspects of system (1.1), especially concerning the Cauchy problem, see e.g. $[7,6]$.

The outline of the paper is as follows. In Section 2, we explain the idea of the proof of Theorem 1, which relies on two main lemmas. We state the key Lemma 2.1 and next use it to prove Lemma 2.2. We then deduce Theorem 1. The proof of Lemma 2.1 is delicate and requires several auxiliary results and preliminaries, which are given in Sections 3 and 4. These sections are respectively devoted to similarity variables and a local criterion for excluding blow-up, and to properties of the ODE system associated with the rescaled equations. Lemma 2.1 is then proved in Section 5. Finally, Theorem 2 is proved in Section 6.

\section{Proof of Theorem 1}

In order to explain the new ideas of our proof, let us first recall that the method in [9] (extending an idea from [10] in the scalar case) is to consider the auxiliary functions

$$
G(r, t)=u_{r}+\varepsilon r^{k} u^{\gamma}, \quad J(r, t)=v_{r}+\varepsilon r^{l} v^{\delta}
$$

(for suitable $\gamma, \delta, k, l>1$ ) and to show that $G, J \leq 0$ on $[0, R] \times[\tau, T$ ) for some $\tau \in(0, T)$. By integrating these inequalities, one then obtains upper bounds on $u, v$, away from $r=0$, hence in particular single-point blow-up. To deduce $G, J \leq 0$ from the maximum principle, one must show that $(G, J)$ satisfies a suitable system of parabolic inequalities. However, the computations reveal that this requires good comparison properties between the two components $u$ and $v$ on $[0, R] \times[\tau, T)$. In [9], such properties could only be established under the very restrictive condition that $p=q$. 
As a starting point of our improvement, we argue by contradiction, assuming that blow-up fails to be localized at the origin, that is,

$$
\limsup _{t \rightarrow T}\left(u\left(r_{0}, t\right)+v\left(r_{0}, t\right)\right)=\infty
$$

for some $r_{0} \in(0, R)$. We then assign ourselves the task of establishing comparison of the components, but only for such solutions, and only in a neighborhood of $x=0$, say $r \leq r_{0} / 2$, having in mind the possibility (due to $[11,3]$ in the case of scalar problems) of localizing the definition of $G$ and $J$ (cf. (2.4)-(2.5) below).

It turns out that under assumption (2.1), one can prove that $(u, v)$ will behave in a neighborhood of $r=0$ like a solution of the corresponding ODE system, which yields very precise comparison properties between $u$ and $v$. Namely, we have the following key lemma.

Lemma 2.1. Let $p, q>1$ and $\Omega=B_{R}$. Let $(u, v)$ be a radially symmetric, nonnegative classical solution of (1.1) such that $u_{r}, v_{r} \leq 0$ and $T<\infty$. Assume that $(u, v)$ satisfies the upper estimates (1.3). If (2.1) holds for some $r_{0} \in(0, R)$, then for all $0 \leq r<r_{0}$ we have

$$
\lim _{t \rightarrow T}(T-t)^{\alpha} u(r, t)=A_{0}, \quad \lim _{t \rightarrow T}(T-t)^{\beta} v(r, t)=B_{0},
$$

where

$$
A_{0}=\left(\beta^{p} \alpha\right)^{1 /(p q-1)}, \quad B_{0}=\left(\alpha^{q} \beta\right)^{1 /(p q-1)} .
$$

In particular,

$$
\lim _{t \rightarrow T}\left[\frac{u^{p+1}}{v^{q+1}}\right](r, t)=D:=A_{0}^{p+1} B_{0}^{-(q+1)}, \quad \text { uniformly on }[0, a] \text { for each } a \in\left(0, r_{0}\right) \text {. }
$$

Lemma 2.1 will be proved in Section 5. The proof is delicate and requires a number of preliminaries and of auxiliary results that will be given in Sections 3 and 4. It will take essential advantage of the radial monotonicity of solutions, in order to identify suitable space limits of rescaled solutions in terms of the corresponding ODE system, and of a criterion for excluding blow-up at a given point, adapted from [1].

Now getting back to the maximum principle approach, we consider the following modified $G, J$ functions:

$$
G(r, t)=u_{r}+\varepsilon c(r) v^{\gamma}, \quad J(r, t)=v_{r}+\varepsilon d(r) u^{\delta},
$$

with

$$
c(r)=\sin ^{2}(\pi r / a), \quad d=K c,
$$

where $\gamma, \delta>1$ and $\varepsilon, K, a>0$ will be chosen later on. Note that $G, J \in C([0, R] \times$ $(0, T)) \cap C^{2,1}((0, R) \times(0, T))$. Assuming Lemma 2.1 is proved, we shall establish: 
Lemma 2.2. Let $(u, v)$ satisfy the assumptions of Lemma 2.1 and let $a=r_{0} / 2$. There exist $\gamma, \delta>1, K>0$ and $\tau \in(0, T)$ such that, for all $\varepsilon \in(0,1], G$ and $J$ satisfy

$$
\begin{cases}G_{t}-G_{r r}-\frac{n-1}{r} G_{r}+\frac{n-1}{r^{2}} G \leq v^{p-1} J, & 0<r \leq a, \tau<t<T, \\ J_{t}-J_{r r}-\frac{n-1}{r} J_{r}+\frac{n-1}{r^{2}} J \leq u^{q-1} G, & 0<r \leq a, \tau<t<T .\end{cases}
$$

Proof of Lemma 2.2. Putting $F=v^{\gamma}$, we compute

$$
G_{t}-G_{r r}=\left(u_{t}-u_{r r}\right)_{r}+\varepsilon\left[c\left(F_{t}-F_{r r}\right)-2 c^{\prime} F_{r}-c^{\prime \prime} F\right]
$$

Using

$$
\begin{gathered}
\left(u_{t}-u_{r r}\right)_{r}=\left(\frac{n-1}{r} u_{r}+v^{p}\right)_{r}=\frac{n-1}{r} u_{r r}-\frac{n-1}{r^{2}} u_{r}+p v^{p-1} v_{r}, \\
F_{t}=\gamma v^{\gamma-1} v_{t}, \quad F_{r}=\gamma v^{\gamma-1} v_{r}, \quad F_{r r} \geq \gamma v^{\gamma-1} v_{r r},
\end{gathered}
$$

hence

$$
F_{t}-F_{r r} \leq \gamma v^{\gamma-1}\left(v_{t}-v_{r r}\right)=\gamma v^{\gamma-1}\left(\frac{n-1}{r} v_{r}+u^{q}\right),
$$

and $u_{r}=G-\varepsilon c v^{\gamma}, v_{r}=J-\varepsilon d u^{\delta}$, we deduce that

$$
\begin{aligned}
G_{t}-G_{r r} \leq & \frac{n-1}{r}\left(G-\varepsilon c v^{\gamma}\right)_{r}-\frac{n-1}{r^{2}}\left(G-\varepsilon c v^{\gamma}\right)+p v^{p-1}\left(J-\varepsilon d u^{\delta}\right) \\
& +\varepsilon v^{\gamma-1}\left[\gamma c\left(\frac{n-1}{r} v_{r}+u^{q}\right)-2 \gamma c^{\prime} v_{r}-c^{\prime \prime} v\right] \\
\leq & \frac{n-1}{r} G_{r}-\varepsilon \frac{n-1}{r} c^{\prime} v^{\gamma}-\frac{n-1}{r^{2}} G+\varepsilon \frac{n-1}{r^{2}} c v^{\gamma}+p v^{p-1}\left(J-\varepsilon d u^{\delta}\right) \\
& +\varepsilon v^{\gamma-1}\left[\gamma c u^{q}-2 \gamma c^{\prime}\left(J-\varepsilon d u^{\delta}\right)-c^{\prime \prime} v\right] .
\end{aligned}
$$

Consequently,

$$
G_{t}-G_{r r}-\frac{n-1}{r} G_{r}+\frac{n-1}{r^{2}} G \leq\left(p v^{p-1}-2 \varepsilon \gamma v^{\gamma-1} c^{\prime}\right) J+\varepsilon H_{1},
$$

where

$$
H_{1}:=-p d v^{p-1} u^{\delta}+v^{\gamma-1}\left[\gamma c u^{q}+2 \varepsilon \gamma d c^{\prime} u^{\delta}+v\left(\frac{n-1}{r}\left(\frac{c}{r}-c^{\prime}\right)-c^{\prime \prime}\right)\right] .
$$

We have

$$
\begin{aligned}
\tilde{H}_{1} & :=\frac{H_{1}}{c v^{\gamma-1} u^{q}} \\
& =-p K \frac{v^{p-\gamma}}{u^{q-\delta}}+\gamma+2 \varepsilon \gamma K c^{\prime} u^{\delta-q}+v u^{-q}\left(\frac{n-1}{r}\left(\frac{1}{r}-\frac{c^{\prime}}{c}\right)-\frac{c^{\prime \prime}}{c}\right) .
\end{aligned}
$$


Symmetrically, exchanging the roles of $u$ and $v$ (and of $c, \gamma$ and $d, \delta$ ), we obtain

$$
J_{t}-J_{r r}-\frac{n-1}{r} J_{r}+\frac{n-1}{r^{2}} J \leq\left(q u^{p q-1}-2 \varepsilon \delta u^{\delta-1} d^{\prime}\right) G+\varepsilon H_{2},
$$

where

$$
\begin{aligned}
\tilde{H}_{2} & :=\frac{H_{2}}{d u^{\delta-1} v^{p}} \\
& :=-\frac{q}{K} \frac{u^{q-\delta}}{v^{p-\gamma}}+\delta+2 \varepsilon \delta \frac{d^{\prime}}{K} v^{\gamma-p}+u v^{-p}\left(\frac{n-1}{r}\left(\frac{1}{r}-\frac{c^{\prime}}{c}\right)-\frac{c^{\prime \prime}}{c}\right) .
\end{aligned}
$$

Assume without loss of generality that $p \geq q$ and choose $\gamma, \delta$ such that

$$
1<\gamma<p, \quad 1<\delta<q
$$

and

$$
p-\gamma=\frac{p+1}{q+1}(q-\delta) \quad\left(\text { i.e. } \gamma=\frac{\delta(p+1)+p-q}{q+1}\right)
$$

Observe that these conditions are satisfied for $\delta>1$ close to 1 , since $\frac{(p+1)+p-q}{q+1}<p$. Using (2.3) we deduce that

$$
\frac{v^{p-\gamma}}{u^{q-\delta}}=\left(\frac{v^{(p+1) /(q+1)}}{u}\right)^{q-\delta} \rightarrow D^{\delta-q} \quad \text { as } t \rightarrow T \text {, uniformly for } r \in[0, a] .
$$

Next set $K=D^{\delta-q}$; it follows from (2.11) that

$$
\gamma<p K D^{\delta-q} \text { and } \delta<\frac{q}{K} D^{q-\delta}
$$

On the other hand, it is easy to check that the function $c$ given in (2.5) satisfies

$$
\frac{n-1}{r}\left(\frac{1}{r}-\frac{c^{\prime}}{c}\right)-\frac{c^{\prime \prime}}{c} \leq C_{1}, \quad 0<r<a .
$$

Combining (2.8), (2.10), (2.12) and (2.13), we see that there exist $\eta>0$ and $\tau$ close to $T$ such that, for any $0<\varepsilon \leq 1$,

$$
\tilde{H}_{1} \leq-\eta+C_{2} u^{\delta-q}+C_{1} v u^{-q} \text { and } \quad \tilde{H}_{2} \leq-\eta+C_{2} v^{\gamma-p}+C_{1} u v^{-p}
$$

for $0<r<a$ and $\tau<t<T$. Since $\delta<q, \gamma<p, q>(q+1) /(p+1)$ and $p>(p+1) /(q+1)$, this along with (2.2) and (2.3) implies that

$$
\tilde{H}_{1}, \tilde{H}_{2} \leq 0, \quad 0<r<a, \tau<t<T
$$

(on taking $\tau$ closer to $T$ if necessary). Going back to (2.7), (2.9), and using $\delta<q, \gamma<p$ and (2.2) again, we obtain the lemma. 
With Lemma 2.1 at hand, we can now conclude the

Proof of Theorem 1. Let $(u, v)$ satisfy the hypotheses of the theorem and assume for contradiction that (2.1) holds for some $r_{0} \in(0, R)$. Let then $G, J$ and $\tau$ be given by Lemma 2.2, for some $\varepsilon \in(0,1]$ to be fixed. By using the maximum principle as in [10], we get $u_{r}, v_{r}<0$ in $(0, R] \times(0, T)$ and $u_{r r}(0, t), v_{r r}(0, t)<0$ in $(0, T)$. Taking $\varepsilon \in(0,1]$ sufficiently small, we deduce that $G(r, \tau), J(r, \tau) \leq 0$ in $[0, a]$ and, by construction, we have $G(r, t)=J(r, t)=0$ for $r=0, a$ and $t \in(\tau, T)$. In view of (2.6), we deduce from the maximum principle (see Remark 2.1) that

$$
G, J \leq 0, \quad 0 \leq r \leq a, \tau<t<T .
$$

On the other hand, (2.3) implies that

$$
C_{3} u^{(p+1) /(q+1)} \leq v \leq C_{4} u^{(p+1) /(q+1)}, \quad 0 \leq r \leq a, \tau_{1} \leq t<T,
$$

for some $\tau_{1} \in(\tau, T)$. Assume $p \leq q$ without loss of generality the first inequality in (2.14) implies that

$$
-u_{r} \geq \varepsilon c(r) v^{\beta} \geq C_{4}^{\beta} \varepsilon c(r) u^{m}, \quad m=\frac{p+1}{q+1} \beta>1 .
$$

By integration, it follows that

$$
u^{1-m}(r, t) \geq C_{5} \int_{0}^{r} c(s) d s, \quad 0 \leq r \leq a, \tau_{1} \leq t<T,
$$

which, along with (2.15), yields

$$
\limsup _{t \rightarrow T}(u(r, t)+v(r, t))<\infty, \quad 0<r \leq a,
$$

a contradiction.

Remark 2.1. The maximum principle for system (2.6) can be derived as follows. Fix $T_{1} \in(\tau, T)$. Multiplying the first inequality in (2.6) by $r^{n-1} G_{+} \geq 0$, integrating by parts over $(0, a)$ and using $G(0, t)=G(a, t)=0$, we obtain

$$
\begin{aligned}
\frac{1}{2} \frac{d}{d t} \int_{0}^{a} r^{n-1} G_{+}^{2} d r & \leq \int_{0}^{a} r^{n-1} v^{p-1} J_{+} G_{+} d r-\int_{0}^{a} \chi_{\{G>0\}} r^{n-1} G_{r}^{2} d r \\
& \leq C \int_{0}^{a} r^{n-1}\left(J_{+}^{2}+G_{+}^{2}\right) d r
\end{aligned}
$$

for $t \in\left[\tau, T_{1}\right]$, and similarly

$$
\frac{1}{2} \frac{d}{d t} \int_{0}^{a} r^{n-1} J_{+}^{2} d r \leq C \int_{0}^{a} r^{n-1}\left(J_{+}^{2}+G_{+}^{2}\right) d r
$$

Adding up, integrating and using $G(\cdot, \tau), J(\cdot, \tau) \leq 0$, we conclude that $G, J \leq 0$ in $[0, a] \times[\tau, T)$. 


\section{Similarity variables and local criterion for excluding blow-up}

In this section, we allow $\Omega$ to be an arbitrary (possibly unbounded) smooth domain of $\mathbb{R}^{n}$. We also allow sign-changing solutions of (1.1). Let $b \in \Omega$. Following [14, 1], we define the (backward) similarity variables around $(T, b)$ by

$$
s=-\log (T-t), \quad y=\frac{x-b}{\sqrt{T-t}}=e^{s / 2}(x-b),
$$

and the rescaled solution by

$$
\left\{\begin{array}{l}
w=w_{b}(y, s)=(T-t)^{\alpha} u(x, t) \\
z=z_{b}(y, s)=(T-t)^{\beta} v(x, t)
\end{array}\right.
$$

on

$$
D:=\left\{(y, s) ; b+y e^{s / 2} \in \Omega, s_{0}<s<\infty\right\}, \quad s_{0}=-\log T .
$$

Note that $D \supset B\left(0, \delta e^{s / 2}\right) \times\{s\}$, with $\delta=\operatorname{dist}(b, \partial \Omega)$. In similarity variables, the differential equations in system (1.1) read

$$
\begin{cases}w_{s}-\mathcal{L} w=z^{p}-\alpha w, & (y, s) \in D \\ z_{s}-\mathcal{L} z=w^{q}-\beta z, & (y, s) \in D\end{cases}
$$

where

$$
\mathcal{L}=\Delta-\frac{y}{2} \cdot \nabla=K^{-1} \nabla \cdot(K \nabla), \quad K(y)=(4 \pi)^{-n / 2} e^{-|y|^{2} / 4}
$$

We denote by $(T(s))_{s \geq 0}$ the semigroup associated with $\mathcal{L}$. More precisely, for each $\phi \in$ $L^{\infty}\left(\mathbb{R}^{n}\right)$, we set $T(s) \phi:=w(\cdot, s)$, where $w$ is the unique classical solution of

$$
\begin{cases}w_{s}=\mathcal{L} w, & y \in \mathbb{R}^{n}, s>0, \\ w(0, y)=\phi(y), & y \in \mathbb{R}^{n} .\end{cases}
$$

For any $\phi \in L^{\infty}\left(\mathbb{R}^{n}\right)$, we put

$$
\|\phi\|_{L_{K}^{r}}=\left(\int_{\mathbb{R}^{n}}|\phi(y)|^{r} K(y) d y\right)^{1 / r}, \quad 1 \leq r<\infty .
$$

Note that

$$
\|\cdot\|_{L_{K}^{p}} \leq C(p, r, n)\|\cdot\|_{L_{K}^{r}}, \quad 1 \leq p<r<\infty .
$$

If the function $\phi$ is defined only on a subdomain of $\mathbb{R}^{n}$, then $\|\phi\|_{L_{K}^{r}}$ denotes the norm of the extension of $\phi$ by 0 . The following lemma (cf. [16, 17]) gives some important properties of $(T(s))_{s \geq 0}$.

Lemma 3.1. (i) For all $1 \leq r \leq \infty$,

$$
\|T(s) \phi\|_{L_{K}^{r}} \leq\|\phi\|_{L_{K}^{r}}, \quad s \geq 0, \phi \in L^{\infty}\left(\mathbb{R}^{n}\right) .
$$

(ii) (Delayed regularizing effect) For all $1 \leq m<r<\infty$, there exist $C_{0}, s^{*}>0$ such that

$$
\|T(s) \phi\|_{L_{K}^{r}} \leq C_{0}\|\phi\|_{L_{K}^{m}}, \quad s \geq s^{*}, \phi \in L^{\infty}\left(\mathbb{R}^{n}\right) .
$$


Remark 3.1. In Section 5 , it will be sometimes useful to rescale the solution written in radial variable. Namely, assume $\Omega=B_{R}, u, v$ radially symmetric, and write $u(x, t)=$ $U(r, t), v(x, t)=V(r, t), r=|x|$. We set

$$
\left\{\begin{array}{l}
W=W_{b}(\rho, s)=(T-t)^{\alpha} U(r, t) \\
Z=Z_{b}(\rho, s)=(T-t)^{\beta} V(r, t) \\
\rho=\frac{r-|b|}{\sqrt{T-t}}=e^{s / 2}(r-|b|)
\end{array}\right.
$$

defined on

$$
\tilde{D}:=\left\{(\rho, s) ;-|b| e^{s / 2}<\rho<(R-|b|) e^{s / 2}, s_{0}<s<\infty\right\}, \quad s_{0}=-\log T .
$$

The equations for $(W, Z)$ are then

$$
\begin{cases}W_{s}-W_{\rho \rho}+\frac{\rho}{2} W_{\rho}=|Z|^{p-1} Z-\alpha W+\frac{(n-1) e^{-s / 2}}{|b|+e^{-s / 2} \rho} W_{\rho}, & (\rho, s) \in \tilde{D} \\ Z_{s}-Z_{\rho \rho}+\frac{\rho}{2} Z_{\rho}=|W|^{q-1} W-\beta Z+\frac{(n-1) e^{-s / 2}}{|b|+e^{-s / 2} \rho} Z_{\rho}, \quad(\rho, s) \in \tilde{D}\end{cases}
$$

We also note that

$$
w_{b}(y, s)=W_{b}\left(\left|b e^{s / 2}+y\right|-|b| e^{s / 2}, s\right)
$$

and a similar relation holds for $z_{b}$.

The main objective of this section is a criterion for excluding blow-up at a given point. A result of this nature was first obtained for a single equation in [15], by using the variational structure (weighted energy) available in the scalar case. In the case of system (1.1), where the energy structure is not available, the authors of [1] were able to obtain such a result, for $\Omega=\mathbb{R}^{n}$, with the help of the smoothing effect in Lemma 3.1. We extend their result to general domains.

Proposition 3.2. Let $M>0$ and let $(u, v)$ be a classical solution of (1.1) satisfying $T<\infty$ and

$$
(T-t)^{\alpha}\|u(t)\|_{\infty} \leq M, \quad(T-t)^{\beta}\|v(t)\|_{\infty} \leq M, \quad 0<t<T
$$

Let $a \in \Omega$ and let $(w, z)$ be the rescaled solution by similarity variables around $(T, a)$. There exists $\varepsilon=\varepsilon(n, p, q, M)>0$ such that, if

$$
\|w(\sigma)\|_{L_{K}^{1}}+\|z(\sigma)\|_{L_{K}^{1}}<\varepsilon
$$

for some $\sigma \geq s_{0}$, then a is not a blow-up point of $(u, v)$, i.e. $(u, v)$ is uniformly bounded in the neighborhood of $(a, T)$. 
Proof. For given $s_{1} \geq s_{0}$, denote respectively by $\bar{w}$ and $\bar{z}$ the solutions of

$$
\begin{cases}\bar{w}_{s}-\mathcal{L} \bar{w}=|\tilde{z}|^{p}-\alpha \bar{w}, & y \in \mathbb{R}^{n}, s>s_{1}, \\ \bar{w}\left(y, s_{1}\right)=\left|\tilde{w}\left(y, s_{1}\right)\right|, & y \in \mathbb{R}^{n},\end{cases}
$$

and

$$
\begin{cases}\bar{z}_{s}-\mathcal{L} \bar{z}=|\tilde{w}|^{q}-\beta \bar{z}, & y \in \mathbb{R}^{n}, s>s_{1}, \\ \bar{z}\left(y, s_{1}\right)=\left|\tilde{z}\left(y, s_{1}\right)\right|, & y \in \mathbb{R}^{n},\end{cases}
$$

where $\tilde{w}(\cdot, s), \tilde{z}(\cdot, s)$ denote the extensions of $w(\cdot, s), z(\cdot, s)$ by 0 to the whole of $\mathbb{R}^{n}$. Clearly $\bar{w}, \bar{z}$ exist globally and, by the maximum principle, we have $|\tilde{w}| \leq \bar{w},|\tilde{z}| \leq \bar{z}$ for $s>s_{1}$. (In order to avoid any difficulty related to the application of the maximum principle in a time dependent domain, just observe that, converting back to $(x, t)$ variables, the comparison can be done in $\Omega$.) By the variation of constants formula, we deduce that

$$
\begin{aligned}
& e^{\alpha s}\left|\tilde{w}\left(s_{1}+s\right)\right| \leq e^{\alpha s} \bar{w}\left(s_{1}+s\right) \\
& \quad \leq T(s)\left|\tilde{w}\left(s_{1}\right)\right|+\int_{0}^{s} e^{\alpha \tau} T(s-\tau)\left|\tilde{z}\left(s_{1}+\tau\right)\right|^{p} d \tau, \quad s_{1} \geq s_{0}, s>0
\end{aligned}
$$

and

$$
e^{\beta s}\left|\tilde{z}\left(s_{1}+s\right)\right| \leq T(s)\left|\tilde{z}\left(s_{1}\right)\right|+\int_{0}^{s} e^{\beta \tau} T(s-\tau)\left|\tilde{w}\left(s_{1}+\tau\right)\right|^{q} d \tau, \quad s_{1} \geq s_{0}, s>0 .
$$

On the other hand, since $\|w(s)\|_{\infty},\|z(s)\|_{\infty} \leq M$ for $s \geq s_{0}$, due to (3.6), the function $h:=\bar{w}+\bar{z}$ satisfies

$$
h_{s}-\mathcal{L} h \leq C_{1} h,
$$

where $C_{1}=\max \left(M^{p-1}, M^{q-1}\right)$. Therefore

$$
\left|\tilde{w}\left(s_{1}+s\right)\right|+\left|\tilde{z}\left(s_{1}+s\right)\right| \leq e^{C_{1} s} T(s)\left(\left|\tilde{w}\left(s_{1}\right)\right|+\left|\tilde{z}\left(s_{1}\right)\right|\right), \quad s_{1} \geq s_{0}, s>0 .
$$

Fix $r>\max (1, n / 2) \max (p, q)$ and let $s^{*}$ be given by Lemma 3.1(ii) with $m=1$. It follows from (3.7), (3.10), and Lemma 3.1(i) that

$$
\|\tilde{w}(\sigma+s)\|_{L_{K}^{1}}+\|\tilde{z}(\sigma+s)\|_{L_{K}^{1}} \leq C_{2} \varepsilon, \quad 0<s \leq s^{*}, \quad \text { with } C_{2}:=e^{C_{1} s^{*}} .
$$

Let now

$T_{0}=\sup \left\{s>0 ; e^{\alpha \tau}\left\|\tilde{w}\left(\sigma+s^{*}+\tau\right)\right\|_{L_{K}^{1}}+e^{\beta \tau}\left\|\tilde{z}\left(\sigma+s^{*}+\tau\right)\right\|_{L_{K}^{1}} \leq 2 C_{2} \varepsilon, \tau \in[0, s]\right\}$.

Note that $T_{0}>0$ and suppose for contradiction that $T_{0}<\infty$. Assuming $p \leq q$ without loss of generality, hence $\alpha \leq \beta$, we have, by (3.12) and (3.11),

$$
\left\|\tilde{w}\left(\sigma+s^{*}+s\right)\right\|_{L_{K}^{1}}+\left\|\tilde{z}\left(\sigma+s^{*}+s\right)\right\|_{L_{K}^{1}} \leq 2 C_{2} \varepsilon e^{-\alpha s}, \quad-s^{*} \leq s \leq T_{0} .
$$


For $0 \leq \tau \leq T_{0}$, (3.10), (3.3), and (3.13) imply

$$
\begin{aligned}
\left\|\tilde{w}\left(\sigma+s^{*}+\tau\right)\right\|_{L_{K}^{r}}+\| \tilde{z}(\sigma & \left.+s^{*}+\tau\right) \|_{L_{K}^{r}} \\
& \leq e^{C_{1} s^{*}}\left(\left\|T\left(s^{*}\right) \tilde{w}(\sigma+\tau)\right\|_{L_{K}^{r}}+\left\|T\left(s^{*}\right) \tilde{z}(\sigma+\tau)\right\|_{L_{K}^{r}}\right) \\
& \leq C_{0} e^{C_{1} s^{*}}\left(\|\tilde{w}(\sigma+\tau)\|_{L_{K}^{1}}+\|\tilde{z}(\sigma+\tau)\|_{L_{K}^{1}}\right) \\
& \leq 2 C_{2} C_{0} e^{C_{1} s^{*}} \varepsilon e^{-\alpha\left(\tau-s^{*}\right)}=C_{3} \varepsilon e^{-\alpha \tau}
\end{aligned}
$$

with $C_{3}=2 C_{2} C_{0} e^{\left(C_{1}+\alpha\right) s^{*}}$. Using (3.8) with $s_{1}=\sigma+s^{*}$, (3.2), (3.1) and (3.14), we deduce that

$$
\begin{aligned}
e^{\alpha s}\left\|\tilde{w}\left(\sigma+s^{*}+s\right)\right\|_{L_{K}^{1}} & \leq\left\|\tilde{w}\left(\sigma+s^{*}\right)\right\|_{L_{K}^{1}}+\int_{0}^{s} e^{\alpha \tau}\left\|\tilde{z}\left(\sigma+s^{*}+\tau\right)\right\|_{L_{K}^{p}}^{p} d \tau \\
& \leq\left\|\tilde{w}\left(\sigma+s^{*}\right)\right\|_{L_{K}^{1}}+C\left(C_{3} \varepsilon\right)^{p} \int_{0}^{s} e^{-\alpha(p-1) \tau} d \tau \\
& \leq\left\|\tilde{w}\left(\sigma+s^{*}\right)\right\|_{L_{K}^{1}}+C C_{3}^{p}(\alpha(p-1))^{-1} \varepsilon^{p} .
\end{aligned}
$$

Similarly, using (3.9) and noting that $\alpha q>\beta$, we obtain

$$
\begin{aligned}
e^{\beta s}\left\|\tilde{z}\left(\sigma+s^{*}+s\right)\right\|_{L_{K}^{1}} & \leq\left\|\tilde{z}\left(\sigma+s^{*}\right)\right\|_{L_{K}^{1}}+\int_{0}^{s} e^{\beta \tau}\left\|\tilde{w}\left(\sigma+s^{*}+\tau\right)\right\|_{L_{K}^{q}}^{q} d \tau \\
& \leq\left\|\tilde{z}\left(\sigma+s^{*}\right)\right\|_{L_{K}^{1}}+C C_{3}^{q}(\alpha q-\beta)^{-1} \varepsilon^{q} .
\end{aligned}
$$

Adding (3.15) and (3.16) for $s=T_{0}$, and using (3.11) for $s=s^{*}$, it follows that

$$
2 C_{2} \varepsilon \leq C_{2} \varepsilon+C C_{3}^{p}(\alpha(p-1))^{-1} \varepsilon^{p}+C C_{3}^{q}(\alpha q-\beta)^{-1} \varepsilon^{q},
$$

hence

$$
C_{2} \leq C(n, p, q, r, M)\left(\varepsilon^{p-1}+\varepsilon^{q-1}\right),
$$

which is impossible for $\varepsilon=\varepsilon(n, p, q, r, M)>0$ sufficiently small. Consequently, we have $T_{0}=\infty$. It follows in particular from (3.14) that

$$
\|\tilde{w}(s)\|_{L_{K}^{r}} \leq C_{4} \varepsilon e^{-\alpha s}, \quad s \geq \sigma+s^{*},
$$

with $C_{4}=C_{3} e^{\alpha\left(\sigma+s^{*}\right)}$.

Now, by continuity, there exists $\eta>0$ small such that (3.7), and hence (3.17), is still true when the point $a$ is replaced by any $b \in \Omega$ such that $|b-a|<\eta$ (note that $\varepsilon$ and $C_{4}$ are independent of $a$ ). Restated in terms of $u$, this means that

$$
\int_{\mathbb{R}^{n}}|u(b+y \sqrt{T-t}, t)|^{r} e^{-|y|^{2} / 4} d y \leq C, \quad T-\delta<t<T,|b-a|<\eta,
$$

for some $\delta \in(0, T)$. Assuming $\delta<\eta / 2$, it follows from Fubini's theorem that

$$
\int_{|z-a|<\eta / 2}|u(z, t)|^{r} d z \leq e^{1 / 4} \int_{|y|<1} \int_{|b-a|<\eta}|u(b+y \sqrt{T-t}, t)|^{r} e^{-|y|^{2} / 4} d y \leq C
$$

for $T-\delta<t<T$. In view of $r>n q / 2$, applying standard local parabolic regularity properties to the second (and next to the first) equation in (1.1), we deduce that $v$ (and next $u$ ) is bounded near $(a, T)$. The proposition is proved. 


\section{Properties of the rescaled ODE system}

In this section, we study nonnegative solutions of the ODE system

$$
\left\{\begin{array}{l}
w^{\prime}=z^{p}-\alpha w \\
z^{\prime}=w^{q}-\beta z
\end{array}\right.
$$

with $p, q>1$ and $\alpha, \beta>0$ (not necessarily given by (1.2)), where' $=d / d s$. In particular, the classification of entire solutions of (4.1) (i.e., global for all $s \in \mathbb{R}$ ) is essential in the proof of Theorem 1. It is clear that the only constant nonnegative solutions of (4.1) are given by $(w, z)=(0,0)$ and $(w, z)=\left(A_{0}, B_{0}\right)$, where

$$
A_{0}=\left(\beta^{p} \alpha\right)^{1 /(p q-1)}, \quad B_{0}=\left(\alpha^{q} \beta\right)^{1 /(p q-1)} .
$$

Proposition 4.1. (a) Let $s_{0} \in \mathbb{R}$ and let $(w, z)$ be a global nonnegative solution of (4.1) for all $s \geq s_{0}$. Then one of the following holds:

(i) $(w, z) \equiv(0,0)$;

(ii) $(w, z) \equiv\left(A_{0}, B_{0}\right)$;

(iii) $w^{\prime} z^{\prime}<0$ for all $s \geq s_{0}$ and $\lim _{s \rightarrow \infty}(w(s), z(s))=\left(A_{0}, B_{0}\right)$;

(iv) there exists $\bar{s} \geq s_{0}$ such that $w^{\prime} z^{\prime}<0$ on $\left[s_{0}, \bar{s}\right)$ and $w^{\prime}, z^{\prime}<0$ on $(\bar{s}, \infty)$. Moreover, $\lim _{s \rightarrow \infty}(w(s), z(s))=(0,0)$.

(b) Let $(w, z)$ be a nonnegative solution of (4.1) with maximal existence interval I. Assume that, for some $s_{0} \in I$,

$$
w\left(s_{0}\right) \geq A_{0}, \quad z\left(s_{0}\right) \geq B_{0}, \quad \text { and } \quad\left(w\left(s_{0}\right), z\left(s_{0}\right)\right) \neq\left(A_{0}, B_{0}\right) .
$$

Then $T:=\sup I<\infty$ and $\lim _{s \rightarrow T^{-}}(w(s)+z(s))=\infty$.

(c) Let $(w, z)$ be a global nonnegative solution of (4.1) for all $s \in \mathbb{R}$. Then either:

(i) $(w, z) \equiv(0,0)$;

(ii) $(w, z) \equiv\left(A_{0}, B_{0}\right)$; or

(iii) $\lim _{s \rightarrow-\infty}(w(s), z(s))=\left(A_{0}, B_{0}\right)$ and $\lim _{s \rightarrow \infty}(w(s), z(s))=(0,0)$.

Proof of (a). Step 1. Assume without loss of generality that $p \geq q>1$. We first note that $(w+z)^{\prime}=w^{q}+z^{p}-(\alpha w+\beta z)$, hence

$$
(w+z)^{\prime} \geq \begin{cases}w^{q}+z^{q}-C(w+z) & \text { if } z \geq 1 \\ w^{q}+z^{q}-1-C(w+z) & \text { if } z \leq 1\end{cases}
$$

In all cases, we have $(w+z)^{\prime} \geq C_{1}(w+z)^{q}-C_{2}$. Consequently, since $(w, z)$ exists on $\left[s_{0}, \infty\right)$, we get

$$
w+z \leq M:=\left(C_{2} / C_{1}\right)^{1 / q}, \quad s \leq s_{0} .
$$

Step 2. Let $\mathcal{R}=[0, \infty)^{2} \backslash\left\{(0,0) ;\left(A_{0}, B_{0}\right)\right\}$. We claim that the regions

$$
\mathcal{R}_{1}=\left\{(X, Y) \in \mathcal{R} ; Y^{p}-\alpha X \geq 0, X^{q}-\beta Y \geq 0\right\}
$$


and

$$
\mathcal{R}_{2}=\left\{(X, Y) \in \mathcal{R} ; Y^{p}-\alpha X \leq 0, X^{q}-\beta Y \leq 0\right\}
$$

are positively invariant for system (4.1).

Indeed, if $(w, z)$ leaves $\mathcal{R}_{1}$ at some time $s_{1} \geq s_{0}$, then either $w^{\prime}=z^{p}-\alpha w=0$, $z^{\prime}=w^{q}-\beta z>0$, or $w^{\prime}=z^{p}-\alpha w>0, z^{\prime}=w^{q}-\beta z=0$ at that time (simultaneous equalities are excluded by local uniqueness). In particular, this implies $w\left(s_{1}\right), z\left(s_{1}\right)>0$. But then $w^{\prime \prime}\left(s_{1}\right)=\left(p z^{p-1} z^{\prime}-\alpha w^{\prime}\right)\left(s_{1}\right)>0$ or $z^{\prime \prime}\left(s_{1}\right)=\left(q w^{q-1} w^{\prime}-\beta z^{\prime}\right)\left(s_{1}\right)>0$, respectively. Consequently, $(w(s), z(s)) \in \mathcal{R}_{1}$ for $s-s_{1}>0$ small; a contradiction. The argument for $\mathcal{R}_{2}$ is similar.

Step 3. By Step 2, if $(w, z)$ is nonconstant, then we have either:

1. $(w(s), z(s)) \in \mathcal{R} \backslash\left(\mathcal{R}_{1} \cup \mathcal{R}_{2}\right)$, i.e.: $w^{\prime} z^{\prime}<0$, for all $s \geq s_{0}$;

2. there exists a first $\bar{s} \geq s_{0}$ such that $(w(\bar{s}), z(\bar{s})) \in \mathcal{R}_{1}$, hence $w^{\prime}, z^{\prime}>0$ on $(\bar{s}, \infty)$ and $w^{\prime} z^{\prime}<0$ on $\left[s_{0}, \bar{s}\right)$; or

3. there exists a first $\bar{s} \geq s_{0}$ such that $(w(\bar{s}), z(\bar{s})) \in \mathcal{R}_{2}$, hence $w^{\prime}, z^{\prime}<0$ on $(\bar{s}, \infty)$ and $w^{\prime} z^{\prime}<0$ on $\left[s_{0}, \bar{s}\right)$.

Indeed, the argument of Step 2 shows that if $(w(\bar{s}), z(\bar{s})) \in \mathcal{R}_{1}$, resp. $\mathcal{R}_{2}$, then $(w(s), z(s))$ $\in \operatorname{int}\left(\mathcal{R}_{1}\right)$, resp. $\operatorname{int}\left(\mathcal{R}_{2}\right)$, for all $s>\bar{s}$.

In case 1 , either $w^{\prime}>0, z^{\prime}<0$, or $w^{\prime}<0, z^{\prime}>0$, for all $s \geq s_{0}$. Since $(w, z)$ is bounded due to $(4.2),(w, z)$ must converge to a (nonzero) equilibrium. This yields assertion (iv). In cases 2 and $3, w$ and $z$ must again converge to an equilibrium. Since $\mathcal{R}_{2} \subset\left\{X \leq A_{0}, Y \leq B_{0}\right\} \backslash\left\{\left(A_{0}, B_{0}\right)\right\}$, the only possibility in case 3 is $(0,0)$. Finally, case 2 cannot occur, due to $\mathcal{R}_{1} \subset\left\{X \geq A_{0}, Y \geq B_{0}\right\} \backslash\left\{\left(A_{0}, B_{0}\right)\right\}$. The proof of statement (a) is complete.

Proof of $(b)$. We claim that the region

$$
\mathcal{R}_{3}=\left\{(X, Y) \in \mathcal{R} ; X \geq A_{0}, Y \geq B_{0}\right\}
$$

is positively invariant for system (4.1). Indeed, if $(w, z)$ leaves $\mathcal{R}_{3}$ at some time $s_{1} \geq s_{0}$, then either $w>A_{0}, z=B_{0}$, or $w=A_{0}, z>B_{0}$ at that time. Therefore either $z^{\prime}=$ $w^{q}-\beta z>A_{0}^{q}-B_{0}=0$, or $w^{\prime}=z^{p}-\alpha w>B_{0}^{p}-A_{0}=0$, hence $(w(s), z(s)) \in \mathcal{R}_{3}$ for $s-s_{1}>0$ small; a contradiction. Hence $(w(s), z(s)) \in \mathcal{R}_{3}$ for all $s \in\left[s_{0}, T\right)$. But this is incompatible with each of assertions (i)-(iv) in part (a). We deduce that $T<\infty$.

Proof of $(c)$. If $(w, z)$ is global on $(-\infty, \infty)$ and nonconstant, then the discussion in Step 3 of part (a) implies that either:

1. $w^{\prime}>0, z^{\prime}<0$ for all $s \in \mathbb{R}$ and $(w, z) \rightarrow\left(A_{0}^{-}, B_{0}^{+}\right)$as $s \rightarrow \infty$;

2. $w^{\prime}<0, z^{\prime}>0$ for all $s \in \mathbb{R}$ and $(w, z) \rightarrow\left(A_{0}^{+}, B_{0}^{-}\right)$as $s \rightarrow \infty$; or

3. there exists $\bar{s} \in \mathbb{R}$ such that $w^{\prime} z^{\prime}<0$ on $(-\infty, \bar{s}), w^{\prime}, z^{\prime}<0$ on $(\bar{s}, \infty)$, and $\lim _{s \rightarrow \infty}(w(s), z(s))=(0,0)$.

In all cases, since $(w, z)$ is bounded on $\mathbb{R}$ due to (4.2), and monotone as $s \rightarrow-\infty$, it must converge to an equilibrium as $s \rightarrow-\infty$, either $(0,0)$ or $\left(A_{0}, B_{0}\right)$. In cases 1 and 2 , both limits are clearly impossible. We are thus left with case 3 and we just need to rule 
out $(0,0)$. To do so, observe that if $w(s)+z(s) \leq \varepsilon$ for some $\varepsilon=\varepsilon(p, q)>0$ small enough, then $(w+z)^{\prime}(s) \leq \varepsilon^{p-1} z+\varepsilon^{q-1} w-\alpha z-\beta w<0$, hence $\sup _{\tau \geq s}(w+z)(\tau) \leq$ $(w+z)(s)$. Therefore, $\lim _{s \rightarrow-\infty}(w, z)=(0,0)$ would imply $w \equiv z \equiv 0$. We conclude that $\lim _{s \rightarrow-\infty}(w, z)=\left(A_{0}, B_{0}\right)$, and this completes the proof of part (c).

We shall also need the following consequence of Proposition 4.1(b), concerning the system of differential inequalities corresponding to (4.1).

Proposition 4.2. Let $s_{0} \in \mathbb{R}$ and let $w, z \in C^{1}\left(\left[s_{0}, T\right)\right), w, z \geq 0$, satisfy

$$
\begin{cases}w^{\prime} \geq z^{p}-\alpha w, & s_{0}<s<T \\ z^{\prime} \geq w^{q}-\beta z, & s_{0}<s<T\end{cases}
$$

along with

$$
w\left(s_{0}\right) \geq A_{0}, \quad z\left(s_{0}\right) \geq B_{0}, \quad \text { and } \quad\left(w\left(s_{0}\right), z\left(s_{0}\right)\right) \neq\left(A_{0}, B_{0}\right) .
$$

Then $T<\infty$.

Proof. Let $(\underline{w}, z)$ be the unique maximal solution of (4.1) such that $\underline{w}\left(s_{0}\right)=w\left(s_{0}\right)$ and $z\left(s_{0}\right)=z\left(s_{0}\right)$, and denote by $s_{1}$ its maximal existence time on the right. It follows from a simple comparison argument that $w \geq \underline{w}$ and $z \geq \underline{z}$ on $\left(s_{0}, \min \left(s_{1}, T\right)\right)$. The conclusion then follows from Proposition 4.1(b).

\section{Proof of Lemma 2.1}

Let $(u, v)$ be a radially symmetric, nonnegative classical solution of (1.1) such that $u_{r}, v_{r}$ $\leq 0$ and $T<\infty$. We assume that $(u, v)$ satisfies the upper estimates (1.3) and that (2.1) holds for some $r_{0} \in(0, R)$. We shall successively prove (2.2) in the cases $r \in\left(0, r_{0}\right)$ and $r=0$ (the former case being used in the proof of the latter).

Step 1. Proof of (2.2) for $r=|a| \in\left(0, r_{0}\right)$.

Let $(W, Z)=\left(W_{a}, Z_{a}\right)$ be the radial rescaling of $(u, v)$ by similarity variables around $(a, T)$ (cf. Remark 3.1). Fix a sequence $s_{j} \rightarrow \infty$. By (1.3), the functions $W$ and $Z$ are bounded. By (3.4) and parabolic estimates, it follows that for some subsequence (still denoted $\left.s_{j}\right)$, the sequence of translates $\left(W_{j}, Z_{j}\right)$ defined by

$$
W_{j}:=W\left(\rho, s+s_{j}\right), \quad Z_{j}:=Z\left(\rho, s+s_{j}\right)
$$

converges in $W^{2,1 ; q}(Q)$ to some pair of functions $(\phi, \psi)$ for each compact $Q \subset \mathbb{R} \times \mathbb{R}$ and each $q \in(1, \infty)$. (Note that the domain of $\left(W_{j}, Z_{j}\right)$ contains $Q$ for $j$ large enough.) Consequently, $(\phi, \psi)$ is a bounded nonnegative solution of

$$
\begin{cases}\phi_{s}-\phi_{\rho \rho}+\frac{\rho}{2} \phi_{\rho}=\psi^{p}-\alpha \phi, & \rho, s \in \mathbb{R}, \\ \psi_{s}-\psi_{\rho \rho}+\frac{\rho}{2} \psi_{\rho}=\phi^{q}-\beta \psi, & \rho, s \in \mathbb{R} .\end{cases}
$$

Moreover, since $u_{r}, v_{r} \leq 0$ on $[0, R) \times(0, T)$, hence

$$
W_{\rho}, Z_{\rho} \leq 0, \quad s_{0}<s<\infty,-a e^{s / 2}<\rho<(R-a) e^{s / 2},
$$


we have

$$
\phi_{\rho}, \psi_{\rho} \leq 0, \quad \rho, s \in \mathbb{R}
$$

Therefore, we may define

$$
\phi_{ \pm}(s)=\lim _{\rho \rightarrow \pm \infty} \phi(\rho, s), \quad \psi_{ \pm}(s)=\lim _{\rho \rightarrow \pm \infty} \psi(\rho, s),
$$

and it is clear that

$$
\phi_{+} \leq \phi_{-}, \quad \psi_{+} \leq \psi_{-} .
$$

We claim that $\phi_{ \pm}, \psi_{ \pm}$satisfy

$$
\begin{cases}\phi_{ \pm}^{\prime}=\psi_{ \pm}^{p}-\alpha \phi_{ \pm}, & s \in \mathbb{R} \\ \psi_{ \pm}^{\prime}=\phi_{ \pm}^{q}-\beta \psi_{ \pm}, & s \in \mathbb{R}\end{cases}
$$

To prove the claim, we shall apply a standard argument based on multiplication by test functions. However, in order to avoid dealing with the (potentially troublesome) terms $(\rho / 2) \phi_{\rho},(\rho / 2) \psi_{\rho}$ in the passage to the limit, it is convenient not to work in the current similarity variables. Thus put

$$
\left\{\begin{array}{l}
U(x, t):=(T-t)^{-\alpha} \phi\left(\frac{x}{\sqrt{T-t}},-\log (T-t)\right), \\
V(x, t):=(T-t)^{-\beta} \psi\left(\frac{x}{\sqrt{T-t}},-\log (T-t)\right),
\end{array}\right.
$$

for $x \in \mathbb{R}$ and $-\infty<t<T$, and observe that

$$
U_{ \pm}(t):=\lim _{x \rightarrow \pm \infty} U(x, t)=(T-t)^{-\alpha} \phi_{ \pm}(-\log (T-t))
$$

and similarly for $V_{ \pm}$. Then $(U, V)$ solves the system (cf. the beginning of Section 3 )

$$
\begin{cases}U_{t}-U_{x x}=V^{p}, & x \in \mathbb{R},-\infty<t<T, \\ V_{t}-V_{x x}=U^{q}, & x \in \mathbb{R},-\infty<t<T .\end{cases}
$$

Fix $\chi \in \mathcal{D}(\mathbb{R})$ with $\int_{\mathbb{R}} \chi=1$ and let $\xi \in \mathcal{D}(-\infty, T)$. For each positive integer $j$, replacing $x$ by $x+j$ and multiplying the first equation in (5.6) by $\chi(x) \xi(t)$, we have

$$
\int_{-\infty}^{T} \int_{\mathbb{R}} U_{t}(x+j, t) \chi(x) \xi(t) d x d t=\int_{-\infty}^{T} \int_{\mathbb{R}}\left[V^{p}+U_{x x}\right](x+j, t) \chi(x) \xi(t) d x d t .
$$

Integrating by parts, we get

$$
\begin{aligned}
-A_{j} \equiv & -\int_{-\infty}^{T} \int_{\mathbb{R}} U(x+j, t) \chi(x) \xi^{\prime}(t) d x d t \\
= & \int_{-\infty}^{T} \int_{\mathbb{R}} V^{p}(x+j, t) \chi(x) \xi(t) d x d t \\
& +\int_{-\infty}^{T} \int_{\mathbb{R}} U(x+j, t) \chi^{\prime \prime}(x) \xi(t) d x d t \equiv B_{j}+C_{j} .
\end{aligned}
$$


By dominated convergence, using the boundedness of $\phi, \psi$ and (5.4), we obtain

$$
\begin{aligned}
\lim _{j \rightarrow \infty} A_{j} & =\int_{-\infty}^{T}\left(\int_{\mathbb{R}} \chi(x) d x\right) U_{+}(t) \xi^{\prime}(t) d t=\int_{-\infty}^{T} U_{+}(s) \xi^{\prime}(t) d t, \\
\lim _{j \rightarrow \infty} B_{j} & =\int_{-\infty}^{T} V_{+}^{p}(t) \xi(t) d t \\
\lim _{j \rightarrow \infty} C_{j} & =\int_{-\infty}^{T}\left(\int_{\mathbb{R}} \chi^{\prime \prime}(x) d x\right) U_{+}(t) \xi(t) d t=0 .
\end{aligned}
$$

Combining (5.7)-(5.10), it follows that $U_{+}^{\prime}=V_{+}^{p}$, and similarly $V_{+}^{\prime}=U_{+}^{q}$, on $(-\infty, T)$ in the distribution sense, hence in the classical sense (since $U_{+}, V_{+} \in L_{\text {loc }}^{\infty}(-\infty, T)$ ). By the same argument, we obtain $U_{-}^{\prime}=V_{-}^{p}, V_{-}^{\prime}=U_{-}^{q}$. Converting back to $\phi_{ \pm}, \psi_{ \pm}$via formula (5.5), we obtain claim (5.3).

Now, in view of Proposition 4.1(c), only two cases are possible.

Case I: $\left(\phi_{+}, \psi_{+}\right) \equiv\left(A_{0}, B_{0}\right)$. Then $\left(\phi_{-}, \psi_{-}\right) \equiv\left(A_{0}, B_{0}\right)$, hence $(\phi, \psi) \equiv\left(A_{0}, B_{0}\right)$.

Case II: $\lim _{s \rightarrow \infty}\left(\phi_{+}(s), \psi_{+}(s)\right)=(0,0)$.

If we can rule out Case II, then for all $t_{j} \rightarrow T$, there exists a subsequence such that $\lim _{j}\left(T-t_{j}\right)^{\alpha} u\left(a, t_{j}\right)=\lim _{j} W\left(0, s_{j}\right)=A_{0}$ and $\lim _{j}\left(T-t_{j}\right)^{\beta} v\left(a, t_{j}\right)=\lim _{j} Z\left(0, s_{j}\right)=$ $B_{0}$, hence (2.2) and we are done.

Let us thus assume that Case II occurs and show that this leads to a contradiction. Pick $b$ such that $|a|<|b|<r_{0}$, and let $\left(w_{b}, z_{b}\right)$ and $\left(W_{b}, Z_{b}\right)$ be respectively the rescaling and the radial rescaling of $(u, v)$ by similarity variables around $(b, T)$. We claim that for any $\varepsilon>0$ there exists $\sigma>s_{0}$ such that

$$
\left\|w_{b}(\sigma)\right\|_{L_{K}^{1}}+\left\|z_{b}(\sigma)\right\|_{L_{K}^{1}} \leq \varepsilon .
$$

Let us prove the claim. By assumption, there exists $\bar{s}$ such that

$$
\phi_{+}(\bar{s}), \psi_{+}(\bar{s})<\varepsilon / 8 .
$$

By definition of $\phi_{+}, \psi_{+}$, this implies the existence of $\bar{\rho}>0$ such that

$$
\phi(\bar{\rho}, \bar{s}), \psi(\bar{\rho}, \bar{s})<\varepsilon / 4 .
$$

Consequently, for all large $j$, we have

$$
W_{a}\left(\bar{\rho}, \bar{s}+s_{j}\right), Z_{a}\left(\bar{\rho}, \bar{s}+s_{j}\right)<\varepsilon / 2 .
$$

With $\bar{\rho}_{j}=\bar{\rho}-(b-a) e^{\left(\bar{s}+s_{j}\right) / 2}$, this is equivalent to

$$
W_{b}\left(\bar{\rho}_{j}, \bar{s}+s_{j}\right), Z_{b}\left(\bar{\rho}_{j}, \bar{s}+s_{j}\right)<\varepsilon / 2 .
$$

Recalling from (5.1) that $\partial_{\rho} W, \partial_{\rho} Z \leq 0$ for $s_{0}<s<\infty$ and $-a e^{s / 2}<\rho<(R-a) e^{s / 2}$, and noting that $\bar{\rho}_{j} \geq-b e^{\left(\bar{s}+s_{j}\right) / 2}$, we deduce that

$$
W_{b}\left(\rho, \bar{s}+s_{j}\right), Z_{b}\left(\rho, \bar{s}+s_{j}\right) \leq \varepsilon / 2, \quad \rho \geq \bar{\rho}_{j},
$$


for all large $j$. Therefore, since $\bar{\rho}_{j} \rightarrow-\infty$ as $j \rightarrow \infty$, by using (3.5), $w_{b}, z_{b} \leq C$ and $\int K d y=1$, we obtain (5.11) with $\sigma=\bar{s}+s_{j}$ and $j$ large enough.

By Proposition 3.2, (5.11) implies that $u(b, t)$ and $v(b, t)$ remain bounded as $t \rightarrow T$, contradicting (2.1) and $|b|<r_{0}$. This contradiction concludes the proof of (2.2) in the case $r \in\left(0, r_{0}\right)$.

Step 2. Proof of (2.2) for $r=0$.

By Step 1, we know that

$$
\lim _{t \rightarrow T}(T-t)^{\alpha} u(r, t)=A_{0}, \quad \lim _{t \rightarrow T}(T-t)^{\beta} v(r, t)=B_{0}, \quad 0<r<r_{0} .
$$

Since $u_{r}, v_{r} \leq 0$, it follows that

$$
\liminf _{t \rightarrow T}(T-t)^{\alpha} u(0, t) \geq A_{0}, \quad \liminf _{t \rightarrow T}(T-t)^{\beta} v(0, t) \geq B_{0} .
$$

Assume for contradiction that $\lim \sup _{t \rightarrow T}(T-t)^{\alpha} u(0, t)>A_{0}$, that is,

$$
\lim _{j \rightarrow \infty}\left(T-t_{j}\right)^{\alpha} u\left(0, t_{j}\right)=\ell>A_{0},
$$

for some sequence $t_{j} \rightarrow T$. Let $(w, z)=\left(w_{0}, z_{0}\right)$ be the rescaling of $(u, v)$ by similarity variables around $(0, T)$. Arguing as at the beginning of Step 1, we deduce that (up to a subsequence) $w\left(y, s+s_{j}\right)$ and $z\left(y, s+s_{j}\right)$ converge locally uniformly to a (bounded nonnegative) solution $(\phi, \psi)$ of

$$
\begin{cases}\phi_{s}-\Delta \phi+\frac{y}{2} \cdot \nabla \phi=\psi^{p}-\alpha \phi, & y \in \mathbb{R}^{n}, s \in \mathbb{R}, \\ \psi_{s}-\Delta \psi+\frac{y}{2} \cdot \nabla \psi=\phi^{q}-\beta \psi, & y \in \mathbb{R}^{n}, s \in \mathbb{R},\end{cases}
$$

and, using (2.2) for $0<r<r_{0}, u_{r}, v_{r} \leq 0$ and (5.13), we also obtain

$$
\phi \geq A_{0}, \psi \geq B_{0} \quad \text { in } \mathbb{R}^{n} \times \mathbb{R}, \quad \text { and } \quad \phi(0,0)>A_{0} .
$$

Multiplying (5.14) by $K$ and integrating by parts (this is easily justified by using the Gaussian decay of the weight $K$ ), we obtain

$$
\frac{d}{d s} \int_{\mathbb{R}^{n}} \phi K d y=\int_{\mathbb{R}^{n}} \psi^{p} K d y-\alpha \int_{\mathbb{R}^{n}} \phi K d y
$$

and

$$
\frac{d}{d s} \int_{\mathbb{R}^{n}} \psi K d y=\int_{\mathbb{R}^{n}} \phi^{q} K d y-\beta \int_{\mathbb{R}^{n}} \psi K d y .
$$

Set $f(s)=\int_{\mathbb{R}^{n}} \phi(s) K d y$ and $g(s)=\int_{\mathbb{R}^{n}} \psi(s) K d y$. By using Jensen's inequality, we deduce that

$$
f^{\prime} \geq g^{p}-\alpha f, \quad g^{\prime} \geq f^{q}-\beta \psi, \quad s \in \mathbb{R},
$$

and (5.15) implies that $f(0)>A_{0}, g(0) \geq B_{0}$. This contradicts Proposition 2.2. Consequently, $\limsup _{t \rightarrow T}(T-t)^{\alpha} u(0, t) \leq A_{0}$, and similarly $\lim \sup _{t \rightarrow T}(T-t)^{\beta} v(0, t)$ $\leq B_{0}$. This, along with (5.12), proves (2.2) in the case $r=0$ and completes the proof of Lemma 2.1. 


\section{Proof of Theorem 2}

In this section, $C$ denotes a positive constant which may vary from line to line. We first give the following lemma.

Lemma 6.1. Under the assumptions of Theorem 2,

$$
\|u(t)\|_{\infty} \geq C_{1}(T-t)^{-\alpha}, \quad\|v(t)\|_{\infty} \geq C_{2}(T-t)^{-\beta}, \quad T / 2<t<T,
$$

for some constants $C_{1}, C_{2}>0$ depending on $u, v$.

Similar results are well-known, but we give the proof for completeness.

Proof. Set $U(t)=u(0, t), V(t)=v(0, t)$. Since $u_{t}, v_{t} \geq 0$, we know that (1.3) is satisfied. Moreover, $U(T)=\infty$ (since otherwise $U$, hence $V$, is bounded). It follows from (1.1) that $U^{\prime} \leq V^{p}, V^{\prime} \leq U^{q}$, hence

$$
U^{\prime}(t) \leq\left(V(0)+\int_{0}^{t} U^{q}(s) d s\right)^{p} \leq C\left(\int_{0}^{t} U^{q}(s) d s\right)^{p}, \quad T / 2<t<T .
$$

Multiplying by $U^{q}$ and integrating, we readily obtain

$$
U^{q+1}(t) \leq U^{q+1}(0)+C\left(\int_{0}^{t} U^{q}(s) d s\right)^{p+1}, \quad T / 2<t<T,
$$

hence

$$
U^{q}(t) \leq C\left(\int_{0}^{t} U^{q}(s) d s\right)^{(p+1) q /(q+1)}, \quad T / 2<t<T .
$$

One more integration yields

$$
\int_{0}^{t} U^{q}(s) d s \geq C(T-t)^{-(q+1) /(p q-1)}, \quad T / 2<t<T .
$$

For $T / 2<\tau<t<T$, by using (6.1), the first inequality in (1.3) and $U$ being nondecreasing, we obtain

$C(T-t)^{-(q+1) /(p q-1)} \leq \int_{0}^{\tau} U^{q} d s+\int_{\tau}^{t} U^{q} d s \leq C^{\prime}(T-\tau)^{-(q+1) /(p q-1)}+(t-\tau) U^{q}(t)$.

For $t$ close enough to $T$, taking $\tau=T-\gamma(T-t)$ with $\gamma>\left(2 C^{\prime} / C\right)^{(p q-1) /(q+1)}, \gamma>1$, we get

$$
U(t) \geq(C / 2 \gamma)(T-t)^{-(p+1) /(p q-1)} .
$$

The lower estimate on $V$ follows similarly.

To prove Theorem 2, we now modify an argument from [20] (used there for scalar equations). Since $u_{t} \geq 0$ and $u_{r}, v_{r} \leq 0$, we have

$$
\frac{\partial}{\partial r}\left(\frac{1}{2} u_{r}^{2}+u v^{p}\right)=\left(u_{r r}+v^{p}\right) u_{r}+p u v^{p-1} v_{r}=\left(u_{t}-\frac{n-1}{r} u_{r}\right) u_{r}+p u v^{p-1} v_{r} \leq 0
$$


hence

$$
\left(\frac{1}{2} u_{r}^{2}+u v^{p}\right)(r, t) \leq u v^{p}(0, t), \quad 0<r<R, 0<t<T .
$$

On the other hand, (1.3) and Lemma 6.1 imply that

$$
v(0, t) \leq C u^{(q+1) /(p+1)}(0, t), \quad T / 2<t<T .
$$

Therefore, we get

$$
\left\|u_{r}(t)\right\|_{\infty} \leq C u^{(m+1) / 2}(0, t), \quad T / 2<t<T,
$$

with $m=p(q+1) /(p+1)$.

Next, for $T / 2<t<T$, let $r_{0}(t)$ be such that

$$
u\left(r_{0}(t), t\right)=\frac{1}{2} u(0, t) .
$$

Note that, since $u_{r}<0$ in $(0, R] \times(0, T)$, the implicit function theorem guarantees that $r_{0}(t)$ is unique and is a continuous function of $t$. Since 0 is the only blow-up point by Theorem 1 , and $u(0, t)=\|u(t)\|_{\infty} \rightarrow \infty$ as $t \rightarrow T$, we see that $r_{0}(t) \rightarrow 0$ as $t \rightarrow T$. Now we have

$$
-u_{r} \leq C u^{(m+1) / 2}, \quad 0 \leq r \leq r_{0}(t), T / 2<t<T .
$$

Integrating in $r$ over $\left(0, r_{0}(t)\right)$, we get

$u^{-(m-1) / 2}\left(r_{0}(t), t\right) \leq u^{-(m-1) / 2}(0, t)+C r_{0}(t)=2^{-(m-1) / 2} u^{-(m-1) / 2}\left(r_{0}(t), t\right)+C r_{0}(t)$

hence $u\left(r_{0}(t), t\right) \geq C r_{0}^{-2 /(m-1)}(t)$. Using $u_{t} \geq 0$, it follows that

$$
u\left(r_{0}(t), T\right) \geq C r_{0}^{-2 /(m-1)}(t)=C r_{0}^{-2 \alpha}(t), \quad T / 2<t<T .
$$

Since $r_{0}$ is continuous and $r_{0}(t) \rightarrow 0$ as $t \rightarrow T$, we deduce that the range $r_{0}((T / 2, T))$ contains an interval of the form $(0, \eta)$, hence the estimate on $u$. The estimate on $v$ is obtained similarly.

\section{References}

[1] Andreucci, D., Herrero, M. A., Velázquez, J. J. L.: Liouville theorems and blow up behaviour in semilinear reaction diffusion systems. Ann. Inst. H. Poincaré Anal. Non Linéaire 14, 1-53 (1997) Zbl 0877.35019 MR 1437188

[2] Caristi, G., Mitidieri, E.: Blow-up estimates of positive solutions of a parabolic system. J. Differential Equations 113, 265-271 (1994) Zbl 0807.35066 |MR 1297658

[3] Chen, X.-Y., Matano, H.: Convergence, asymptotic periodicity, and finite-point blow-up in one-dimensional semilinear heat equations. J. Differential Equations 78, 160-190 (1989) Zbl 0692.35013 MR 0986159

[4] Chlebík, M., Fila, M.: From critical exponents to blow-up rates for parabolic problems. Rend. Mat. Appl. (7) 19, 449-470 (1999) Zbl 0980.35057 MR 1789482

[5] Deng, K.: Blow-up rates for parabolic systems. Z. Angew. Math. Phys. 47, 132-143 (1996) Zbl 0854.35054 MR 1408675 
[6] Deng, K., Levine, H. A.: The role of critical exponents in blow-up theorems: the sequel. J. Math. Anal. Appl. 243, 85-126 (2000) Zbl 0942.35025 MR 1742850

[7] Escobedo, M., Herrero, M. A.: Boundedness and blow up for a semilinear reaction-diffusion system. J. Differential Equations 89, 176-202 (1991) Zbl 0735.35013 MR 1088342

[8] Fila, M., Souplet, Ph.: The blow-up rate for semilinear parabolic problems on general domains. NoDEA Nonlinear Differential Equations Appl. 8, 473-480 (2001) Zbl 0993.35046 MR 1867324

[9] Friedman, A., Giga, Y.: A single point blow-up for solutions of semilinear parabolic systems. J. Fac. Sci. Univ. Tokyo Sect. IA Math. 34, 65-79 (1987) Zbl 0648.35042 MR 0882125

[10] Friedman, A., McLeod, B.: Blow-up of positive solutions of semilinear heat equations. Indiana Univ. Math. J. 34, 425-447 (1985) Zbl 0576.35068 MR 0783924

[11] Fujita, H., Chen, Y.-G.: On the set of blow-up points and asymptotic behaviours of blowup solutions to a semilinear parabolic equation. In: Analyse Mathématique et Applications, Contributions en l'honneur de Jacques-Louis Lions, Gauthier-Villars, Paris, 181-201 (1988) Zbl 0732.35008 MR 0956959

[12] Galaktionov, V. A., Kurdyumov, S. P., Samarski1̆, A. A.: A parabolic system of quasilinear equations, I. Differential Equations 19, 2123-2140 (1983) Zbl 0556.35071 MR 0729570

[13] Galaktionov, V. A., Kurdyumov, S. P., Samarskiĭ, A. A.: A parabolic system of quasilinear equations, II. Differential Equations 21, 1544-1559 (1985) Zbl 0599.35085 MR 0807679

[14] Giga, Y., Kohn, R. V.: Asymptotically self-similar blow-up of semilinear heat equations. Comm. Pure Appl. Math. 38, 297-319 (1985) Zbl 0585.35051 MR 0784476

[15] Giga, Y., Kohn, R. V.: Nondegeneracy of blowup for semilinear heat equations. Comm. Pure Appl. Math. 42, 845-884 (1989) Zbl 0703.35020 MR 1003437

[16] Gross, L.: Logarithmic Sobolev inequalities. Amer. J. Math. 97, 1061-1083 (1976) Zbl 0318.46049 MR 0420249

[17] Herrero, M. A., Velázquez, J. J. L.: Blow-up behaviour of one-dimensional semilinear parabolic equations. Ann. Inst. H. Poincaré Anal. Non Linéaire 10, 131-189 (1993) Zbl 0813.35007 MR 1220032

[18] Merle, F.: Solution of a nonlinear heat equation with arbitrarily given blow-up points. Comm. Pure Appl. Math. 45, 263-300 (1992) Zbl 0785.35012 MR 1151268

[19] Quittner, P., Souplet, Ph.: Global existence from single-component $L_{p}$ estimates in a semilinear reaction-diffusion system. Proc. Amer. Math. Soc. 130, 2719-2724 (2002) Zbl 0993.35048 MR 1843418

[20] Souplet, $\mathrm{Ph} .:$ The influence of gradient perturbations on blow-up asymptotics in semilinear parabolic problems: a survey. In: Progr. Nonlinear Differential Equations Appl. 64, Birkhäuser, 473-496 (2005) Zbl 1094.35060 MR 2185234

[21] Souplet, Ph., Tayachi, S.: Blow-up rates for nonlinear heat equations with gradient terms and for parabolic inequalities. Colloq. Math. 88, 135-154 (2001) Zbl 0984.35077 MR 1814920

[22] Velázquez, J. J. L.: Estimates on the (n-1)-dimensional Hausdorff measure of the blow-up set for a semilinear heat equation. Indiana Univ. Math. J. 42, 445-476 (1993) Zbl 0802.35073 MR 1237055

[23] Velázquez, J. J. L.: Blow up for semilinear parabolic equations. In: Recent Advances in Partial Differential Equations, M. A. Herrero and E. Zuazua (eds.), Res. Appl. Math. 30, Masson, Paris, 131-145 (1994) Zbl 0798.35072 MR 1266206

[24] Weissler, F. B.: Single point blow-up for a semilinear initial value problem. J. Differential Equations 55, 204-224 (1984) Zbl 0555.35061 MR 0764124

[25] Zaag, H.: A Liouville theorem and blow-up behavior for a vector-valued nonlinear heat equation with no gradient structure. Comm. Pure Appl. Math. 54, 107-133 (2001) Zbl 1029.35113 MR 1787109 